\title{
Cardiac Juvenile Xanthogranuloma in an Infant Presenting with Pericardial Effusion
}

\author{
Daisuke Kobayashi, MD, ${ }^{*}$ Ralph E. Delius, MD, ${ }^{\dagger}$ Larisa V. Debelenko, MD, $\mathrm{PhD},{ }^{\ddagger}$ and \\ Sanjeev Aggarwal, MD* \\ *Division of Cardiology, Carman and Ann Adams Department of Pediatrics, Children's Hospital of Michigan, Wayne \\ State University School of Medicine, Detroit, Mich, USA; 'Department of Cardiovascular Surgery, Children's Hospital of \\ Michigan, Wayne State University School of Medicine, Detroit, Mich, USA; ҒDepartment of Pathology, Wayne State \\ University, Detroit, Mich, USA
}

\section{A B S T R A C T}

Juvenile xanthogranuloma is a rare histiocytic disorder of childhood mainly affecting skin and rarely deep soft tissues and viscera. We report a 2 -month-old infant who presented with respiratory distress secondary to a large pericardial effusion associated with an epicardial mass. Excisional biopsy was performed and the mass was diagnosed as juvenile xanthogranuloma. The child is well without evidence of disease 8 months following the excision. The corresponding literature on juvenile xanthogranuloma with cardiac manifestations is reviewed.

Key Words. Cardiac Tamponade; Pericardial Effusion; Juvenile Xanthogranuloma

\section{Introduction}

J uvenile xanthogranuloma (JXG) is a rare histiocytic disorder predominantly affecting skin and occurring in the first two decades of life. ${ }^{1}$ Rare extracutaneous and systemic (cutaneous-visceral) forms of JXG have been described., ${ }^{2,3}$ The lesion is thought to originate from dermal dendrocytes, the subtype of histiocytes that is related to but different from Langerhans cells. ${ }^{4}$ The biology of the disease is not clear, but in a majority of cases and in all cases of solitary lesions, the disease does not recur and the lesions may spontaneously regress. Only four cases of JXG involving the heart have been previously reported. ${ }^{5-9}$

\section{Case Report}

This female infant was born at 33 weeks gestation via $\mathrm{C}$-section secondary to intrauterine growth retardation and oligohydramnios. She had stayed in the neonatal intensive care unit for 3 weeks and was discharged home in good health except for anemia of prematurity. Her family history was significant for a 22-month-old brother with a single kidney. At 2 months of age, she presented to the hospital with a respiratory distress for 2 days. Her mother denied fever, cough, or feeding difficulty.

Congenit Heart Dis. 2013;8:E106-E110
On admission, she was tachycardic (heart rate 170 beats per minute) and tachypneic ( 60 breaths per minute). Blood pressure was $81 / 36 \mathrm{~mm} \mathrm{Hg}$ with a temperature of $36.9^{\circ} \mathrm{C}$ and oxygen saturation of $100 \%$ with $0.25 \mathrm{~L} / \mathrm{min}$ oxygen via nasal cannula. She weighed $2.8 \mathrm{~kg}(<5$ th percentile $)$ with a height of $48 \mathrm{~cm}$ ( $<5$ th percentile). On physical examination, she had mild subcostal and intercostal retraction. Lungs were clear to auscultation with bilateral equal air entry. Detailed cardiovascular examination revealed distant heart sounds without an appreciable murmur, pericardial rub, or gallop. The peripheries were well perfused and pulses were normal. There was no peripheral edema or clubbing. A solitary small bright red, slightly raised skin lesion was noted on the left midclavicular area and felt to be consistent with hemangioma. Complete blood count showed white blood cells of $7400 / \mathrm{mm}^{3}$, hemoglobin $7.1 \mathrm{~g} / \mathrm{dL}$, hematocrit of $23.2 \%$ (mean corpuscular volume $91.5 \mathrm{fL}$ and mean corpuscular hemoglobin concentration $30.1 \%$ ), and platelets of $390000 / \mathrm{mm}^{3}$. A chest radiograph showed cardiomegaly and pulmonary congestion. The echocardiogram revealed a moderate- to large-sized pericardial effusion with signs of cardiac tamponade and a tumor in pericardial space (Figure 1). The cardiac tumor measured $2.1 \times 2.4 \mathrm{~cm}$, and was attached to the right ven- 
tricular free wall in the pericardial space. The mass was homogenously mildly hyperechogenic to myocardial tissue without obvious internal cavity or calcified lesions. A pericardial drain was placed under echocardiographic guidance and approximately $40 \mathrm{~mL}$ of red sanguineous pericardial fluid was drained. Analysis of pericardial fluid showed glucose $64 \mathrm{mg} / \mathrm{dL}$, lactate dehydrogenase $720 \mathrm{U} / \mathrm{L}$, protein $29 \mathrm{gm} / \mathrm{dL}$ with nucleated

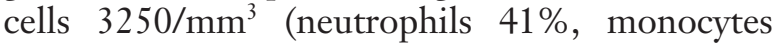
$40 \%$, eosinophils $19 \%$ ), and no red blood cells. The pericardial fluid continued to accumulate and drained approximately $10-20 \mathrm{~mL}$ daily. Cardiac magnetic resonance showed an exophytic soft tissue mass measuring $2.3 \times 2.5 \times 1.3 \mathrm{~cm}$, adherent to the right ventricular outflow tract. It was mildly heterogeneous on $\mathrm{T}_{1}$-weighted images and isointense to minimally hyperintense to the myocardial tissue, and homogeneous and isointense on $\mathrm{T}_{2}$-weighted images (Figure 2). There was no internal fatty tissue, calcification, nor delayed enhancement. An oncology consult was obtained and flow cytometry of pericardial fluid was performed which showed no evidence of leukemia, lymphoma, or neuroblastoma. Cytology of pericardial fluid showed three-dimensional clusters consisting of many eosinophils, macrophages, and large scattered atypical cells, which were arranged in vague fascicles with a fair amount of cytoplasm, admixed with scattered inflammatory cells. There was no immunoreactivity with CD1a, S-100, Berep4, and CD34. Teratoma was considered unlikely based on imaging findings, and, although rare, malignancy could not be excluded. Because of persistent pericardial effusion, the decision was made to perform excisional biopsy.

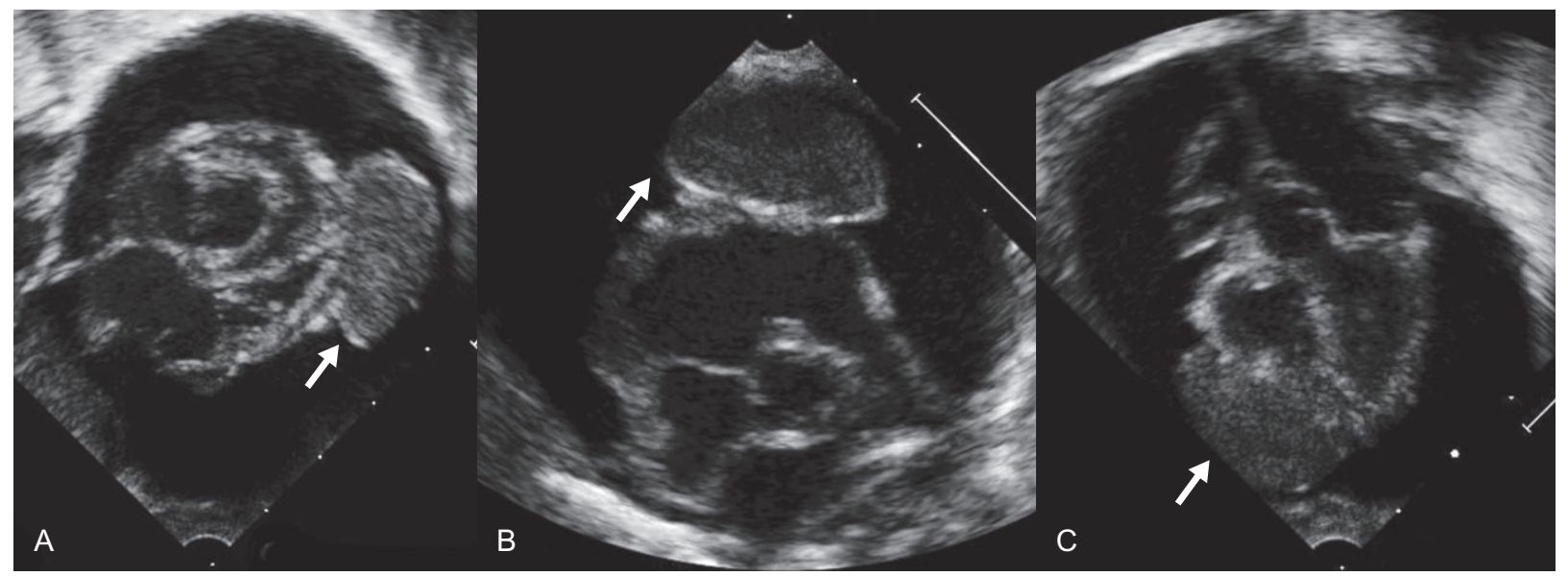

Figure 1. Echocardiography, showing an exophytic homogeneous tumor (arrows) adherent to the right ventricular outflow tract. There is no internal cavity or calcified lesion in the tumor. Note the large pericardial effusion.

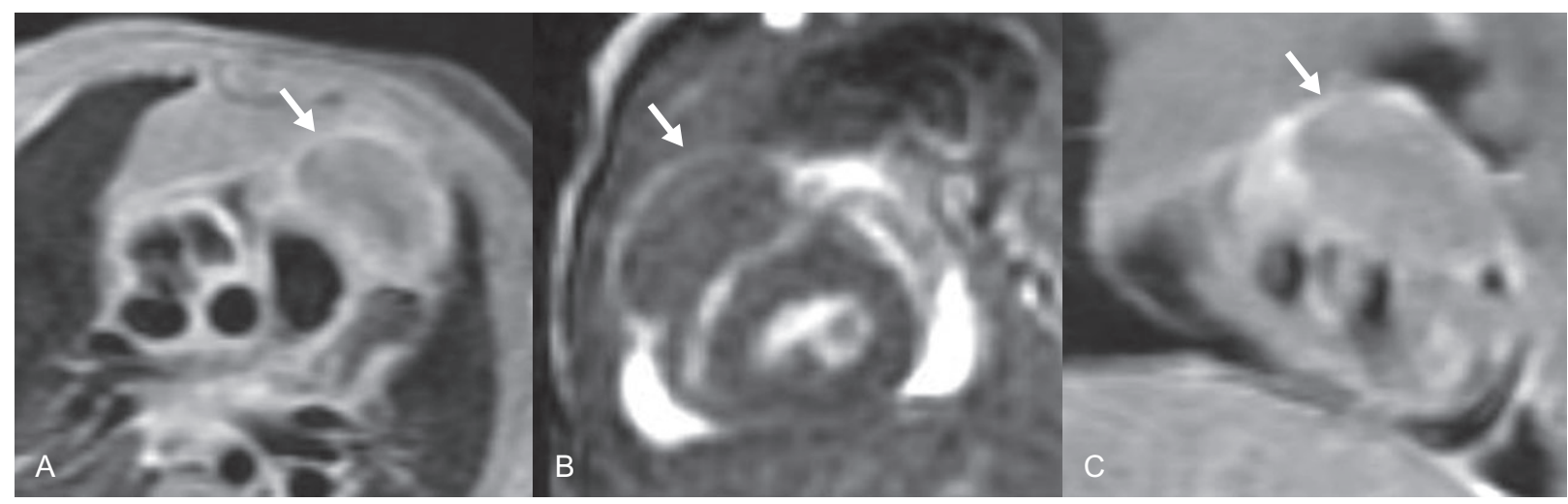

Figure 2. Cardiac MRI, showing an exophytic soft tissue mass (arrows) measuring $2.3 \times 2.5 \times 1.3 \mathrm{~cm}$, adherent to the right ventricular outflow tract. (A) Axial view: no gadolinium enhancement was noted on early phases; (B) sagittal view, tumor is homogeneous and isointense on $\mathrm{T}_{2}$-weighted images; and $(\mathrm{C})$ coronal view: tumor is isointense to minimally hyperintense and mildly heterogeneous on $\mathrm{T}_{1}$-weighted images. 
Intraoperative inspection revealed an exophytic mass on the right ventricular outflow tract, which was extremely friable and cauliflower-like in appearance (Figure 3). The tumor extended within $3 \mathrm{~mm}$ of the pulmonary valve and within $3 \mathrm{~mm}$ of the left anterior descending artery. The tumor was excised and a bovine pericardial patch was used for reconstruction of the right ventricular free wall and outflow tract. Pathology examination showed lesion composed of histiocytes with abundant pink cytoplasm and folded nuclei and admixed Toutontype giant cells, eosinophils, rare lymphocytes, and plasma cells (Figure 4). Xanthomatous cells were not frequent, although hemosiderosis was prominent. No hemophagocytosis, necrosis, or atypical mitoses were observed. The lesional cells were

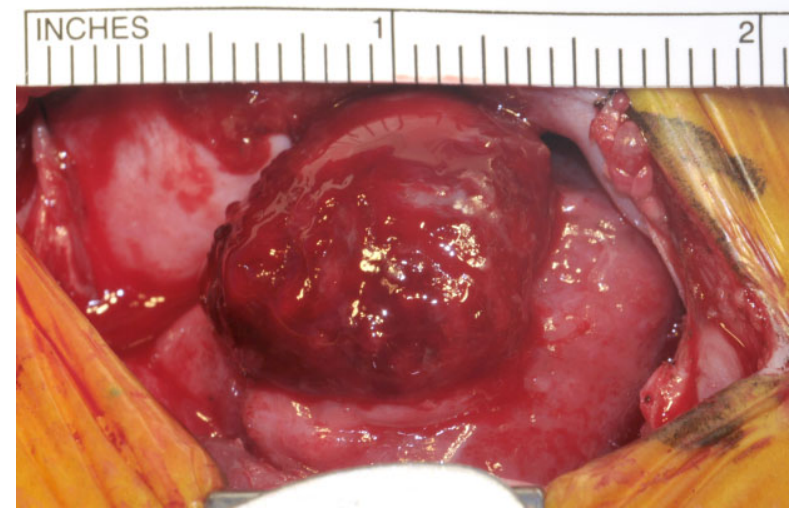

Figure 3. Macroscopic image of lesion. The mass was exophytic on the right ventricular outflow tract, which was extremely friable and resembled a cauliflower. immunoreactive with CD68, lysozyme, and CD31, and negative for CD1a, S-100, CD45, CD34, and GLUT-1. Ki-67 proliferative index was relatively high (approximately 30\%). The lesion showed infiltrative growth to the myocardium (Figure 4A), and resection margins were involved.

Ultrastructurally, the tumor nuclei had mature chromatin and cytoplasm was well-developed and filled with numerous mitochondria, smooth and rough endoplasmic reticulum, free ribosomes, lipid droplets, vacuoles, and occasional tubuloreticular inclusions. The cells exhibited numerous cellular processes, in keeping with histiocytic line of differentiation, and were connected by simple junctions. No Birbeck granules were identified. Cytogenetics showed normal female karyotype, 46,XX. Light and ultrastructural morphology and immunophenotype were diagnostic of JXG involving myocardium.

The postoperative course was uneventful. She was extubated on day 3 and discharged home on day 7 postsurgery. At discharge, echocardiography revealed normal biventricular function without right outflow tract obstruction or pericardial effusion. On the latest 8-month follow-up, she remained asymptomatic with no skin lesions, normal biventricular function, and no pericardial effusion on echocardiography.

\section{Discussion}

In the first and the largest series of 174 JXGs, solitary extracutaneous lesions were reported only in $5 \%$ of all cases; the series contained no cases
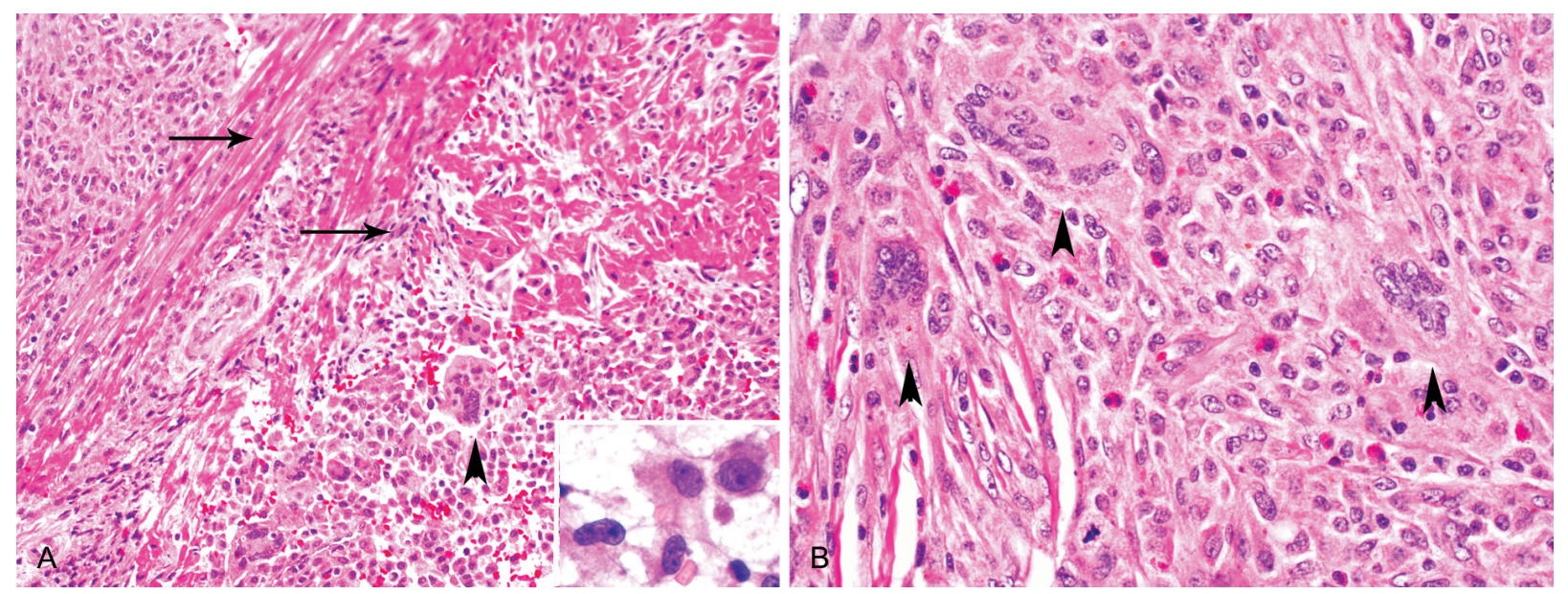

Figure 4. Histopathology. (A) Periphery of the lesion showing infiltrative growth of poorly cohesive histiocytes into surrounding myocardium (arrows). A large Touton giant cell is seen (arrowhead). Hematoxylin \& eosin, $\times 200$. Insert: intraoperative imprint showing lesional cells with large eosinophilic cytoplasm and folded nuclei, hematoxylin \& eosin, $\times 600)$. (B) Center of the lesion with 3 Touton giant cells (arrowheads), hematoxylin \& eosin, $\times 400$. 
of cardiac JXGs. ${ }^{2}$ The subsequent comprehensive series of 129 cases contained only one case of cardiac JXG in a setting of systemic disease. ${ }^{3}$ Three additional cases of cardiac JXG have been reported since then, demonstrating that the heart could be a primary site for isolated visceral or combined visceral-cutaneous forms of JXG ${ }^{5-8} \mathrm{~A}$ summary of the five cardiac JXG cases (including the present one) is presented in Table 1. Overall, four of five patients were females, with a median age of 1 month (range: 1 day-3 months). Two epicardial JXGs presented with cardiac tamponade associated with a large pericardial effusion, requiring pericardial drainage. Two cases of solitary cardiac JXG were successfully treated with surgical resection and did well at follow-up of 5 years and 8 months, respectively. ${ }^{5}$

Our patient presented with a large pericardial effusion associated with epicardial mass. There was neither cutaneous nor other deep soft tissue/ visceral lesions, suggestive of JXG. Flow cytometry and cytology of pericardial effusion was inconclusive but showed the presence of atypical cells. Therefore, malignancy could not be excluded. Excisional biopsy showed unexpected pathological diagnosis of cardiac JXG in our case. Solitary cardiac JXG, especially with epicardial type, is associated with pericardial effusion. Open biopsy should be pursued to treat effusion and establish tissue diagnosis.

The optimal therapy for systemic JXG remains undefined. ${ }^{1}$ The three cases of systemic JXG were treated aggressively (radiation therapy in one and chemotherapy in two). One infant with extensive systemic JXG died at 34 days of age despite chemotherapy, whereas another achieved regression of cardiac and skin lesions after 2 years of chemotherapy. ${ }^{3,8}$

Yeh et al. described computed tomography and magnetic resonance imaging (MRI) characteristics of JXG. ${ }^{8}$ Reported MRI findings of JXG are a well-circumscribed mass with: (1) isointensity as myocardium on $\mathrm{T}_{1}$-weighted images; (2) slight hyperintensity on $\mathrm{T}_{2}$-weighted images; and (3) minimal central enhancement after gadolinium administration. Our MRI findings were concordant with this description. Echocardiographic features of cardiac JXG have not been previously described. Malcić et al. reported intracardiac JXG, as a bulging spherical pedunculated mass. ${ }^{5}$

In a study of 78 pediatric cardiac tumors, a pericardial effusion was associated with teratoma (100\%), fibroma (47\%), rhabdomyoma (21\%), malignancy $(42 \%)$, and hemangioma $(22 \%) .{ }^{10}$ The

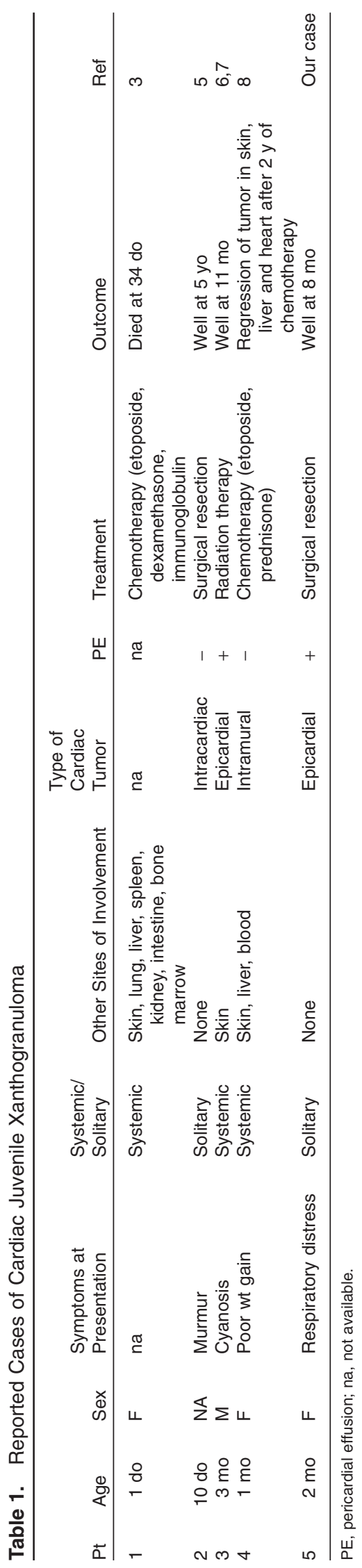

Congenit Heart Dis. 2013;8:E106-E110 
12 cases of malignant tumors were: osteosarcoma 3 , lymphoma 2, rhabdomyosarcoma 2, angiosarcoma 1, B-cell lymphoma 1, desmoplastic round cell tumor 1, melanoma 1 , and medulloblastoma 1 . Malignant tumors most often presented as a solitary lesion $(92 \%)$ at a mean age of 14.5 years (range: 9-18.6 years). Cardiac MRI characteristics of malignant cardiac tumor are invasive appearance and variable heterogeneous intensity. Teratoma is an intrapericardial tumor, invariably associated with pericardial effusion and was the important differential diagnosis in our case. Echocardiographic characteristics of pericardial teratoma are a well-circumscribed hyperechoic solid tumor, with cystic components and foci of calcifications. ${ }^{11}$ Cardiac MRI characteristics of teratoma include: (1) intrapericardial site, often compressing the superior vena cava and/or right atrium; (2) multilocular bosselated mass with solid and cystic areas; and (3) hypointensity on first-pass myocardial perfusion imaging. ${ }^{10}$ Management of pericardial teratoma is early resection to prevent cardiac failure and metastasis of a potentially malignant tumor. ${ }^{11}$ Teratoma usually does not have myocardial involvement and can be removed without cardiac dissection.

In summary, we report the fifth case of cardiac JXG involving the epicardium and superficial myocardium and associated with a large, lifethreatening pericardial effusion. This presentation was raising a question of malignancy. The surgery was both diagnostic by obtaining the tissue for pathologic analysis and curative by subtotally reducing the mass and preventing further pericardial effusions. Despite positive margins, no tumor recurrence was observed in 8 months, in keeping with the benign nature of the lesion and its propensity to spontaneous regression. Echocardiography and cardiac MRI findings of JXG are delineated and differentiated from teratoma.

\section{Conclusion}

Cardiac JXG occurs in infancy as a solitary and systemic disease. The solitary cardiac JXG appears to have a good prognosis after surgical excision. Cardiac JXG should be considered in the differential diagnosis of an epicardial tumor with pericardial effusion.

\section{Author Contribution}

D.K. wrote the manuscript and collected the data for review of the literature. R.E.D., L.V.D., and S.A. critically edited the manuscript and approved it.
Corresponding Author: Daisuke Kobayashi, MD, Division of Cardiology, Carman and Ann Adams Department of Pediatrics, Children's Hospital of Michigan, Wayne State University School of Medicine, 3901 Beaubien Blvd, Detroit, MI 48201-2119, USA. Tel: +1(313)745-5481; Fax: +1(313)993-0894; E-mail: dkobayas@dmc.org

Conflict of interest: No conflict of interest, grants, or other financial support.

Accepted in final form: May 6, 2012.

\section{References}

1 Freyer DR, Kennedy R, Bostrom BC, Kohut G, Dehner LP. Juvenile xanthogranuloma: forms of systemic disease and their clinical implications. f Pediatr. 1996;129:227-237.

2 Dehner LP. Juvenile xanthogranulomas in the first two decades of life: a clinicopathologic study of 174 cases with cutaneous and extracutaneous manifestations. Am F Surg Pathol. 2003;27:579-593.

3 Janssen D, Harms D. Juvenile xanthogranuloma in childhood and adolescence: a clinicopathologic study of 129 patients from the kiel pediatric tumor registry. Am $\mathcal{7}$ Surg Pathol. 2005;29:21-28.

4 Weitzman S, Jaffe R. Uncommon histiocytic disorders: the non-Langerhans cell histiocytoses. Pediatr Blood Cancer. 2005;45:256-264.

5 Malcić I, Novick WM, Dasović-Buljević A, Jelasić D, Jelusić M, Kniewald H. Intracardiac juvenile xanthogranuloma in a newborn. Pediatr Cardiol. 2001;22:150-152.

6 Pois AJ, Johnson LA. Multiple congenital xanthogranulomas of skin and heart. Report of a case. Dis Chest. 1966;50:325-329.

7 Eller JL. Roentgen therapy for visceral juvenile xanthogranuloma, including a case with involvement of the heart. Am 7 Roentgenol Radium Ther Nucl Med. 1965;95:52-55.

8 Yeh BM, Nobrega KT, Reddy GP, Qayyum A. Juvenile xanthogranuloma of the heart and liver: MRI, sonographic, and CT appearance. AfR Am $\mathcal{F}$ Roentgenol. 2007;189:W202-W204.

9 Seo IS, Min KW, Mirkin LD. Juvenile xanthogranuloma. Ultrastructural and immunocytochemical studies. Arch Pathol Lab Med. 1986;110:911-915.

10 Beroukhim RS, Prakash A, Buechel ER, et al. Characterization of cardiac tumors in children by cardiovascular magnetic resonance imaging: a multicenter experience. 7 Am Coll Cardiol. 2011;58: 1044-1054.

11 MacKenzie S, Loken S, Kalia N, et al. Intrapericardial teratoma in the perinatal period. Case report and review of the literature. 7 Pediatr Surg. 2005; 40:e13-e18. 